\title{
A REVIEW OF FLUID FORCES INDUCED BY A CIRCULAR CYLINDER OSCILLATING AT LOW AMPLITUDE AND HIGH FREQUENCY IN CYLINDRICAL CONFINEMENT.
}

\author{
Cedric Leblond \\ Service Technique et Scientifique \\ DCN Propulsion \\ Indret - BP 30 \\ 44620 LA MONTAGNE, FRANCE \\ Email: cedric.leblond@dcn.fr
}

\author{
Jean Francois Sigrist \\ Christian Laine \\ Service Technique et Scientifique \\ DCN Propulsion \\ Indret - BP 30 \\ 44620 LA MONTAGNE, FRANCE
}

\author{
Bruno Auvity \\ Hassan Peerhossaini \\ Laboratoire de Thermocinetique \\ CNRS UMR 6607 \\ Rue C. Pauc - BP 50609 \\ 44306 NANTES, FRANCE
}

\begin{abstract}
This paper deals with fluid forces induced by an oscillating rigid circular cylinder in a fluid initially at rest. The amplitude of the imposed movement is assumed sufficiently small so that no wake is formed. The objective of the present paper is to review different theoretical methods to evaluate fluid forces. A wide variety of conditions is considered, from inviscid, compressible flows in infinite fluid domains, to viscous, incompressible and strongly confined ones. A special care is taken to underline the limits of the simplified models regarding real fluid effects, such as three-dimensional centrifugal instabilities. This review is related to a study whose ultimate aim is to predict dynamic fluid load during a typical shock encountered in the environment of a military ship.
\end{abstract}

\section{INTRODUCTION}

The patterns induced by a bluff body undergoing harmonic oscillation in a direction normal to its axis in a still fluid are of considerable fundamental and engineering interest. If the amplitude of the body is high enough, the boundary layer can break away from the body surface by a phenomenon called separation [1,2]. Vortices are then generated in each half cycle, resulting in different wakes formation $[3,4]$. These vortices can strongly influence the fluid force experienced by the body [5-7]. This paper focuses on the regimes induced by sufficiently small displacements of the body so that no separation occurs in the fluid flow [5]. The study of these regimes have several practical applications. In infinite fluid domains, they can yield to the prediction of the loading on ocean structures exposed to waves. In confined fluid domains, they are specially of interest in fields such as heat exchanger and squeeze film dampers design [8-11].

In case of shock loading, naval components are subjected to high acceleration and frequency motions. These components can be in contact with a viscous fluid. In order to improve design margins and ensure security, long life and satisfactory operating performance of the components, the precise knowledge of the fluid effects on solids is of major importance [12]. In particular, the sources of damping have to be well characterized since they can limit the vibrations of the structures.

A $R \times D$ program has been launched by DCN Propulsion in order to obtain some insights into real flow effects. It consists of studying the flow induced by a circular cylinder moving in a direction normal to its axis in a fluid initially at rest. A first approach was to consider added mass effects in fully coupled fluid-structure problems $[13,14]$. Interest was put on harmonic oscillations in a cylindrically confined fluid. A second approach was to take into account some history effects in an infinite fluid medium surrounding a rigid circular cylinder [15]. The motion of interest was then a displacement induced by an unique sinusoidal period of acceleration imposed to the cylinder. The next steps are to consider complex phenomena such as unsteady separation, inertial effects in lubricant films and three-dimensional 


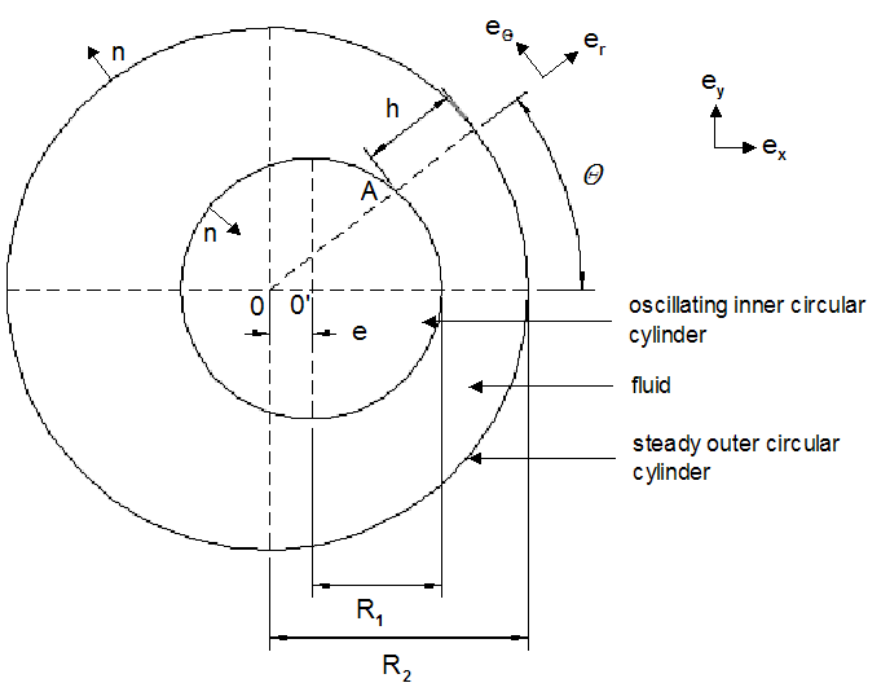

Figure 1. THE GEOMETRICAL CONFIGURATION.

instabilities.

The development of new measuring techniques (Particle Image Velocimetry for example) and the increased hardware performance can now provide detailed information about the time variation of local flow properties. Literal expressions of fluid forces [16-18] can then be used to make the link between these properties and the time-dependent loading of structures. The most convenient expressions are reviewed in the second section. In the section called "Simplified models" some engineer's models of fluid force prediction on moving circular cylinder are exposed. Simple effects such as resonance, added mass and damping are highlighted in the case of attached, laminar and two-dimensional flows. The limits of these simple models and some remaining challenges concerning real flow effects are exposed in the last section. They may be numerically out of reach and difficult to appreciate experimentally. Modeling them has already been a major issue for more than fifty years and still represents a huge challenge for the future. In the following of this introduction, the key parameters of the system considered in this paper are presented.

We consider a viscous fluid of density $\rho$ confined in an annular region, see Fig. 1. The dynamic viscosity is noted $\mu=\rho v$ with $v$ the kinematic viscosity. The speed of sound in the fluid at rest is noted $c$. The inner circular cylinder of radius $R_{1}$ (and diameter $d=2 R_{1}$ ) is subjected to a harmonic motion $e(t)$ in the direction $\mathbf{e}_{\mathbf{x}}$ defined by:

$$
e(t)=e_{m} \cos \omega t
$$

The maximum velocity $V_{m}=e_{m} \omega$ is also used in this paper. The outer cylinder of radius $R_{2}$ is at rest. For the case of an infinite fluid medium, it comes $R_{2}=\infty$. When the fluid is considered as viscous, no-slip boundary conditions are used on the cylinders, however when the fluid is assumed inviscid, only the normal component of the velocity is conserved on the walls and the fluid is allowed to slip. The length of the cylinders are supposed infinite so that end effects may be neglected. Moreover heat transfers and axial flows are not considered in this paper. This study is also restricted to a sufficiently small displacements of the inner circular cylinder so that no wake is formed. There are at least seven independent parameters $\left(R_{1}, R_{2}, e_{m}, \omega, v, \rho, c\right)$ for three units $(k g, m, s)$, so the problem is driven by at least four dimensionless numbers. An overview of all possible fluid states in the whole dimensionless numbers space is out of reach so far. Only some particular behaviors will be given. For instance, only subsonic displacement of the inner cylinder is considered:

$$
M=\frac{V_{m}}{c}<<1
$$

where $M$ is the Mach number. The interesting dimensionless parameters in this problem depend on the assumptions adopted in each model. For an infinite viscous fluid domain, the flow has historically $[5,19]$ been characterised by the following dimensionless numbers:

$$
\begin{aligned}
\beta & =\frac{1}{2 \pi} \frac{d^{2} \omega}{v} \text { the Stokes number } \\
K C & =2 \pi \frac{e_{m}}{d} \text { the Keulegan-Carpenter number }
\end{aligned}
$$

In the case of a confined fluid, the Keulegan-Carpenter number is not sufficient to quantify the inner cylinder displacement. So it is convenient to introduce the instantaneous eccentricity ratio $\varepsilon(t)$ defined by the fraction of the eccentricity $e(t)$ with the radial clearance $C=R_{2}-R_{1}$. The following dimensionless parameter can then be formed [20]:

$$
\varepsilon_{m}=\frac{e_{m}}{R_{1}(\alpha-1)} \text { with } \alpha=\frac{R_{2}}{R_{1}} .
$$

For a compressible inviscid flow, $\beta$ can not be used in order to quantify the frequency of the imposed movement, and the nondimensional frequency $\Omega$ is preferred:

$$
\Omega=\frac{R_{1} \omega}{c} .
$$

Surface state effects may also influence the flow behaviour. They are usually characterized using the non-dimensional number $k / d$ where $k$ is the roughness height. However, this ratio is generally not sufficient and the shape of the asperities have to be considered [21-23].

\section{FORCE EXPRESSIONS Literal fluid forces expressions on a moving body}

There are several ways to evaluate fluid forces experienced by a moving body. An extrinsic measurement yields the forces by the precise knowledge of the body trajectory, as in the case of particle-wall collision [24]. It can be achieved by the use of strain and/or displacement gauges. An intrinsic measurement is inferred from the knowledge of the fluid velocity fields and their 
derivatives [16]. This latter method has considerable advantages. For instance, it does not required any gauges and allows to measure small force level. Different formulae can be used so as to proceed to an intrinsic method. Some of them are given in what follows. By definition the force acting on the body surface $S_{b}(t)$ can be expressed by:

$$
\mathbf{F}(t)=\mathbf{F}_{p}+\mathbf{F}_{\mu}=-\int_{S_{b}(t)}(-p \mathbf{n}+\overline{\bar{T}} \cdot \mathbf{n}) d s
$$

where $\mathbf{F}_{p}$ and $\mathbf{F}_{\mu}$ represent respectively the contribution of the pressure and the viscous forces, $\mathbf{n}$ is the unit normal vector (see Fig. 1) and $\overline{\bar{T}}$ denotes the viscous stress tensor given by $\left.\overline{\bar{T}}=\mu\left(\nabla \mathbf{u}+(\nabla \mathbf{u})^{T}\right)\right)$ with $\mathbf{u}$ the local fluid velocity. Even if Eqn. (7) combines two clearly distinct effects, pressure and viscosity, they are not fully decoupled since the fact that the viscosity is taken into account can strongly modify the pressure field. As a consequence, the pressure force is generally different from the one obtained with an inviscid flow. Since the viscous force is expressed by velocity derivatives at the boundary, the accuracy of its calculation depends on the resolution at the vicinity of the wall. However, in most experiments, and specially in the case of a moving wall, the flow is not well described at this place. To overcome this issue, Eqn. (7) can be expressed more generally in term of time-dependent control volume $V(t)$ bounded externally by the body surface:

$$
\begin{aligned}
\mathbf{F}(t) & =-\frac{d}{d t} \int_{V(t)} \rho \mathbf{u} d V \\
& +\int_{S(t)} \mathbf{n} \cdot\left(-p \overline{\bar{I}}-\rho\left(\mathbf{u}-\dot{e}(t) \mathbf{e}_{\mathbf{x}}\right) \mathbf{u}+\overline{\bar{T}}\right) d s
\end{aligned}
$$

The first term of the right hand side is the time rate change of momentum within the control volume and the second one gathers respectively the instantaneous pressure force, the net flux of momentum and the instantaneous shear force on the control surface. Sometimes, the knowledge of the pressure distribution is missing, so Eqns. $(7,8)$ are particularly inconvenient. This is the case in computations when the hydrodynamic problem is not cast in velocity-pressure form, but in velocity-vorticity or in streamfunction-vorticity formulation. This is also the case in the experiments when the velocity field is obtained by Particle Image Velocimetry (PIV). Then, either pressure is obtained as a solution of a separate problem or it is removed from Eqn. (8). This latter method can be achieved $[17,25]$ but may result in the apparition of a geometrical variable. However, both this new variable and the pressure are not directly visible. So as to provide a straightforward link between the flow patterns and the fluid forces, it can be convenient to reformulate Eqn. (8) with the vorticity field $\boldsymbol{\omega}$ only. In an infinite fluid domain, it yields [18]:

$$
\begin{aligned}
\mathbf{F}(t)= & -\frac{1}{N-1} \rho \frac{d}{d t} \int_{V(t)} \mathbf{x} \times \omega d V \\
& +\frac{1}{N-1} \rho \frac{d}{d t} \int_{S_{b}(t)} \mathbf{x} \times\left(\mathbf{n} \times \dot{e}(t) \mathbf{e}_{\mathbf{x}}\right) d s
\end{aligned}
$$

where $N$ is the spatial dimension and $\mathbf{x}$ the distance from an arbitrary reference point. Knowing the boundary conditions on the body, the second term of the right hand side is fully determined. In our case, the force is expressed by:

$$
F(t)=-\rho \frac{d}{d t} \int_{V(t)} \mathbf{x} \times \omega d V+\rho \pi R_{1}^{2} \ddot{e}(t) \mathbf{e}_{\mathbf{x}}
$$

It is a sum of a vorticity-dependent term and an inviscid inertia term. Unsteadiness of the vorticity located in the wake and in the boundary layer results in unsteadiness of the fluid force. However, as it will be seen in the last section, the first term of the right hand side contains also a part in phase with the acceleration of the body, so that the second term alone can not be identified as the added mass term. Equation (10), which is correct for an infinite fluid domain, can be extended to an arbitrary domain by the control volume approach. A family of relations having the form:

$$
\mathbf{F}(t)=\frac{d}{d t} \int_{V(t)} \mathbf{a}_{1} d V+\int_{S(t)} \mathbf{n} \cdot \mathbf{a}_{2} d s+\frac{d}{d t} \int_{S_{b}(t)} \mathbf{a}_{3} d s
$$

can be found in [16]. $\mathbf{a}_{1}, \mathbf{a}_{2}$ and $\mathbf{a}_{3}$ are functions of the velocity and vorticity fields. The most convenient equation seems to be the "flux equation", which involves surface integrals only, with the added constraint being that the velocity field is divergence free. In our case, it can be written:

$$
\begin{aligned}
\mathbf{F}(t) & =\rho \int_{S(t)} \mathbf{n} \cdot \gamma_{f l u x} d s+\rho \pi R_{1}^{2} \ddot{e}(t) \mathbf{e}_{\mathbf{x}} \text { where } \\
\gamma_{f l u x} & =\frac{1}{2} \mathbf{u}^{2} \overline{\bar{I}}+\mathbf{u u}-\mathbf{u}(\mathbf{x} \times \omega)+\omega(\mathbf{x} \times \mathbf{u}) \\
& -\left[\left(\mathbf{x} \cdot \frac{\partial \mathbf{u}}{\partial t}\right) \overline{\bar{I}}-\mathbf{x} \frac{\partial \mathbf{u}}{\partial t}+\frac{\partial \mathbf{u}}{\partial t} \mathbf{x}\right] \\
& +[\mathbf{x} \cdot(\nabla \cdot \overline{\bar{T}}) \overline{\bar{I}}-\mathbf{x}(\nabla \cdot \overline{\bar{T}})]+\overline{\bar{T}}
\end{aligned}
$$

This equation is convenient in situations where the flow cannot be resolved accurately near the body surface. It seems that the fluid force predictions are all the more accurate as the surface of the control volume is close to the body [26].

\section{Another way of thinking the fluid forces}

In the previous section, literal expressions of fluid forces have been given. So as to make them useful, the knowledge of the velocity or / and vorticity distributions is required. They can be obtained by direct numerical simulation, experimental data or simplified models (see the next section). Fluid forces can also be viewed as general functions of the body displacement, velocity 
and acceleration:

$$
\mathbf{F}(t)=\mathbf{F}(e(t), \dot{e}(t), \ddot{e}(t))
$$

The assumption of small amplitude motions about the reference position $(e(t), \dot{e}(t), \ddot{e}(t))=(0,0,0)$ allows expressing the force as a Taylor Serie Expansion. It comes at the main order:

$$
\begin{gathered}
\mathbf{F}(t)=-\left(K e(t)+C_{v} \dot{e}(t)+M_{a} \ddot{e}(t)\right) \mathbf{e}_{\mathbf{x}} \\
\text { where } K=-\frac{\partial F}{\partial e}, C_{v}=-\frac{\partial F}{\partial \dot{e}} \text { and } M_{a}=-\frac{\partial F}{\partial \ddot{e}}
\end{gathered}
$$

$K$ is called the stiffness coefficient, $C_{V}$ the damping coefficient and $M_{a}$ the added mass. For a quiescent fluid, the fluid stiffness force is null. $M_{a}$ is particularly important when the system is subjected to fast transient motions. It is often written $M_{a}=-\rho \pi R_{1}^{2} C_{a}$ for a circular cylinder, where $C_{a}$ is called the added mass coefficient. So, for a quiescent fluid, the force at the main order is reduced to:

$$
\mathbf{F}(t)=-\left(C_{\mathrm{v}} \dot{e}(t)+\rho \pi R_{1}^{2} C_{a} \ddot{e}(t)\right) \mathbf{e}_{\mathbf{x}}
$$

In case of large displacements, non-linear effects can become important and Eqn. (15) is no more adapted. Some experimental results are nevertheless presented by showing the variation of the coefficients with the motion amplitude. In 1950, Morison et al. [27] proposed a force decomposition for the determination of the in-line force induced by a harmonic motion of a cylindrical body in an infinite domain:

$$
\mathbf{F}(t) \cdot \mathbf{e}_{\mathbf{x}}=-\rho R_{1} C_{d}|\dot{e}(t)| \dot{e}(t)-\rho \pi R_{1}^{2} C_{a} \ddot{e}(t)
$$

The first term of the right hand side is a velocity-squareddependent term, with $C_{d}$ called the drag coefficient, and the second one is the acceleration-dependent inertial force. $C_{d}$ and $C_{a}$ are Fourier-averaged empirical coefficients. This force decomposition is semi-empirical and its justification is strictly pragmatic [28]. It has been experimentally confirmed in finite areas of the parameters space [5].

\section{SIMPLIFIED MODELS}

\section{Incompressible inviscid model}

In this model the fluid is inviscid, and the flow incompressible. The amplitude of the inner cylinder is assumed so small ( $K C<<1$ and $\varepsilon_{m}<<1$ ) that the Navier-Stokes equations can be linearized around a steady state flow. In these conditions, the fluid is only characterized by:

$$
\Delta p(r, t)=0
$$

with the following boundary conditions:

$$
\begin{aligned}
& \nabla p\left(R_{1}, t\right) \cdot \mathbf{n}=-\rho \ddot{e}(t) \mathbf{e}_{\mathbf{x}} \cdot \mathbf{n} \\
& \nabla p\left(R_{2}, t\right) \cdot \mathbf{n}=0
\end{aligned}
$$

where $p$ is the pressure. The solution is found by Fritz [29]:

$$
p(r, \theta, t)=\rho \frac{R_{1}^{2}}{R_{2}^{2}-R_{1}^{2}}\left(r+\frac{R_{2}^{2}}{r}\right) \ddot{e}(t) \cos \theta
$$

In this inviscid case, Eqn. (7) is reduced to:

$$
\mathbf{F}(t)=-\int_{0}^{2 \pi} p\left(R_{1}, \theta\right) R_{1} \cos \theta d \theta \mathbf{e}_{\mathbf{x}}
$$

which gives with the solution Eqn. (20):

$$
\mathbf{F}(t)=-\rho \pi R_{1}^{2} C_{\text {Fritz }} \ddot{e}(t) \mathbf{e}_{\mathbf{x}} \text { where } C_{\text {Fritz }}=\frac{\alpha^{2}+1}{\alpha^{2}-1}
$$

$C_{\text {Fritz }}$ is the added mass coefficient. In an infinite fluid domain, it is reduced to 1 . In this potential flow, fluid force is directly proportional to the body acceleration. When the cylinder is subjected to excitation, both the cylinder and the added fluid mass have to be accelerated without phase difference, as if they were rigidly attached.

\section{Incompressible viscous model}

Under the same assumptions as in the previous section, but considering a viscous fluid, the flow can be described by:

$$
\Delta^{2} \psi-\frac{1}{v} \frac{\partial}{\partial t} \Delta \psi=0
$$

with the no-slip boundary conditions:

$$
\begin{aligned}
& \mathbf{u}\left(R_{1}, t\right)=\dot{e}(t) \mathbf{e}_{\mathbf{x}} \\
& \mathbf{u}\left(R_{2}, t\right)=0 \mathbf{e}_{\mathbf{x}}
\end{aligned}
$$

where $\psi$ is the stream function defined by $\mathbf{u}=\nabla \times \psi$ and $\mathbf{u}$ is the local velocity. The exact solution for a finite domain is given by Chen $[30,31]$. The corresponding force takes the form of Eqn. (15) and is reduced to:

$$
\begin{aligned}
& C_{\mathrm{v}}=2 \pi \mu \sqrt{\pi \beta} \frac{\alpha\left(\alpha^{3}+1\right)}{\left(\alpha^{2}-1\right)^{2}} \\
& C_{a}=\frac{\alpha^{2}+1}{\alpha^{2}-1}+\frac{4}{\sqrt{\pi \beta}}
\end{aligned}
$$

for large $\beta$ [32]. By taking $\beta \rightarrow \infty$ in the above equations, the inviscid model reappears. In contrast to the previous section, the viscous fluid does not respond everywhere instantaneously to the structural motion, resulting in a phase difference between the body and the fluid motions.

In 1851 Stokes [33] presented a solution for an oscillating cylinder in an infinite fluid domain. The oscillations are so small that the flow around the body is assumed laminar, unseparated and stable. The Stokes' solution was extended to higher terms by Wang in 1968 [34], resulting in the so-called Stokes-Wang theory. The corresponding fluid force is often expressed thanks to the Morison representation Eqn. (16). The use of this representation may be misleading since it suggests that the relationship between the force and the instantaneous velocity is non-linear, whereas the theory predicts a linear one. However the expression of damping in terms of a drag coefficient is historically used, expressing the theoretical results in this form by means of the approximation $\cos x|\cos x| \approx 8 /(3 \pi) \cos x$ in Eqn. (16). The core- 


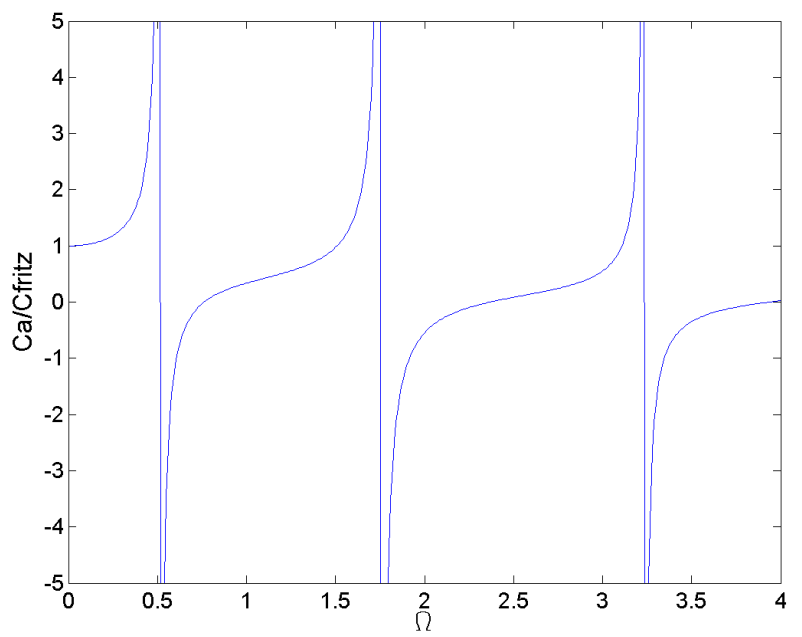

Figure 2. RATIO BETWEEN THE ADDED MASS COEFFICIENT FOR A CIRCULAR CYLINDER INSIDE A COMPRESSIBLE FLUID ANNULUS AND THE FRITZ ONE, AT $\alpha=3$.

sponding coefficients are given by

$$
\begin{aligned}
C_{a} & =1+4(\pi \beta)^{-1 / 2}+(\pi \beta)^{-3 / 2} \\
C_{d} & =\frac{3 \pi^{3}}{2 K C}\left[(\pi \beta)^{-1 / 2}+(\pi \beta)^{-1}-\frac{1}{4}(\pi \beta)^{-3 / 2}\right]
\end{aligned}
$$

The inertia force is modified by the Stokes number, expressing the rate of diffusion. So the fluid force can not be decomposed into an inviscid inertia force, and a viscous force. Both are affected by the diffusion of vorticity in which resides the memory of viscous fluids. At large value of $\beta$, the added mass coefficient tends to the Fritz's coefficient, see Eqn. (22).

\section{Compressible inviscid model}

Compressible effects can arise in a system even if the velocity imposed to the inner cylinder is much smaller than the speed of sound $(M<<1)$. Acoustic resonance may take place at some special frequencies. In order to illustrate this effect, the fluid is assumed inviscid, and the body motion of small amplitude $(K C<<1$ and $\varepsilon<<1)$. The Euler equations can then be linearized resulting in a wave equation for the velocity potential $\Phi$, defined such that $\mathbf{u}=\nabla \Phi$ :

$$
\left(\frac{1}{r} \frac{\partial}{\partial r}\left(r \frac{\partial}{\partial r}\right)+\frac{1}{r^{2}} \frac{\partial^{2}}{\partial \theta^{2}}-\frac{1}{c^{2}} \frac{\partial^{2}}{\partial t^{2}}\right) \Phi=0
$$

By the method of separation of variables and with the boundary conditions:

$$
\begin{aligned}
& \frac{\partial \Phi}{\partial r}\left(R_{1}, t\right)=-V_{m} \sin \omega t \cos \theta \\
& \frac{\partial \Phi}{\partial r}\left(R_{2}, t\right)=0
\end{aligned}
$$

a non-resonant solution can be obtained [31]:

$$
\begin{aligned}
\Phi(r, t) & =\frac{R_{1}}{\Omega} \frac{1}{D(\Omega)}\left[Y^{\prime}(\alpha \Omega) J_{1}\left(\frac{r}{R_{1}} \Omega\right)\right. \\
& \left.-J_{1}^{\prime}(\alpha \Omega) Y_{1}\left(\frac{r}{R_{1}} \Omega\right)\right] \dot{e}(t) \cos \theta
\end{aligned}
$$

where $D(\Omega)=J_{1}^{\prime}(\Omega) Y_{1}^{\prime}(\alpha \Omega)-Y_{1}^{\prime}(\Omega) J_{1}^{\prime}(\alpha \Omega) . J_{1}$ and $Y_{1}$ are respectively the Bessel functions of the first and second kind of order one. The prime denotes the derivative with respect to the function argument. So the fluid force on the inner cylinder can be deduced from:

$$
\mathbf{F}(t)=\rho \int_{0}^{2 \pi} \frac{\partial \Phi}{\partial t}\left(R_{1}, \theta, t\right) R_{1} \cos \theta d \theta \mathbf{e}_{\mathbf{x}}
$$

which takes the form of Eqn. (15) with:

$$
\begin{aligned}
C_{a} & =\frac{1}{\Omega} \frac{1}{D(\Omega)}\left(J_{1}^{\prime}(\alpha \Omega) Y_{1}(\Omega)-Y_{1}^{\prime}(\alpha \Omega) J_{1}(\Omega)\right) \\
C_{\mathrm{V}} & =0
\end{aligned}
$$

The added mass is displayed in Fig. 2 for $\alpha=3$. When $\Omega \rightarrow$ 0 , it tends to the Fritz coefficient (Eqn. (22)). Equation (31) is invalid when the resonant condition $D\left(\Omega_{s}\right)=0$ is satisfied. $\Omega_{s}$ is a non-dimensional resonance frequency defined by $\Omega_{s}=\omega_{s} R_{1} / c$ with $\omega_{s}$ the corresponding dimensional one. Practically, when the frequency of the oscillation is close to a resonance frequency, the response amplitude of the fluid can be quite large even if the amplitude of inner cylinder is small. Figure 3 shows the curve which gives the smallest resonance frequency in function of $\alpha$. Following the methodology used in [35] for another problem, a solution of Eqn. (29) which satisfies the boundary conditions and the resonance condition can be found. Let us write a solution in the form:

$$
\begin{aligned}
\hat{\Phi} & =c \cos \theta \frac{V_{m}}{\omega} \frac{1}{D(\omega)}[E(\omega, \omega) \sin \omega t \\
& \left.-E\left(\omega_{s}, \omega_{s}\right) \sin \omega_{s} t\right] \text { with } \\
E\left(\omega_{1}, \omega_{2}\right) & =Y_{1}\left(\Omega_{1} \frac{r}{R_{1}}\right) J_{1}^{\prime}\left(\alpha \Omega_{2}\right)-J_{1}\left(\Omega_{1} \frac{r}{R_{1}}\right) Y_{1}^{\prime}\left(\alpha \Omega_{2}\right)
\end{aligned}
$$

and $\Omega_{i}$ defined by $\Omega_{i}=\omega R_{1} / c$ (for $i=1,2$ ). $\hat{\Phi}$ is a solution of Eqn. (29). Equation (35) can be rewritten as:

$$
\begin{aligned}
\hat{\Phi} & =c \cos \theta \frac{V_{m}}{\omega}\left(E(\omega, \omega) \frac{\sin \omega t-\sin \omega_{s} t}{D(\omega)}\right. \\
& +\sin \omega_{s} t \frac{E(\omega, \omega)-E\left(\omega_{s}, \omega\right)}{D(\omega)} \\
& \left.+\sin \omega_{s} t \frac{E\left(\omega_{s}, \omega\right)-E\left(\omega_{s}, \omega_{s}\right)}{D(\omega)}\right)
\end{aligned}
$$

Taking the limit $\omega \rightarrow \omega_{s}$ and applying the Hopital's rule to Eqn. (36), give a resonant solution which satisfies the boundary conditions Eqn. (30): 


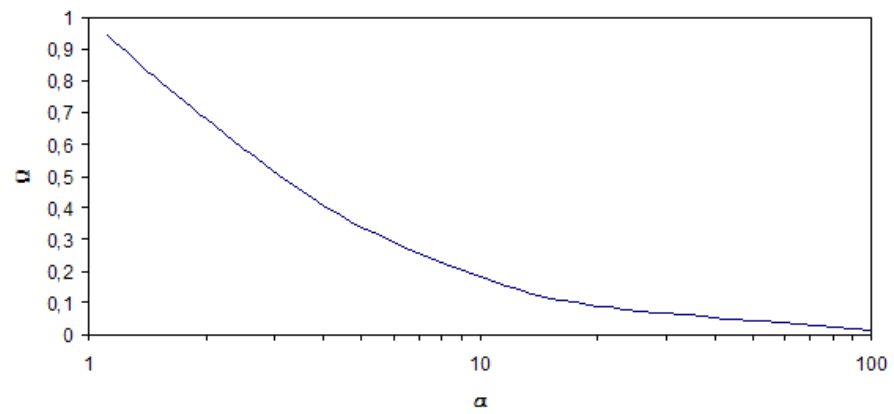

Figure 3. VARIATION OF THE SMALLEST DIMENSIONLESS RESONANT FREQUENCY WITH THE CONFINEMENT RATIO.

$$
\begin{aligned}
\Phi & =\frac{c \cos \theta V_{m}}{\omega_{s}^{2} Z\left(\omega_{s}\right)}\left[\omega_{s} t \cos \omega_{s} t E\left(\omega_{s}, \omega_{s}\right)\right. \\
& +\Omega_{s} \frac{r}{R_{1}} \sin \omega_{s} t\left(Y_{1}^{\prime}\left(\Omega_{s} \frac{r}{R_{1}}\right) J_{1}^{\prime}\left(\alpha \Omega_{s}\right)-J_{1}^{\prime}\left(\Omega_{s} \frac{r}{R_{1}}\right) Y_{1}^{\prime}\left(\alpha \Omega_{s}\right)\right) \\
& \left.+\alpha \omega_{s} \sin \omega_{s} t\left(Y_{1}\left(\Omega_{s} \frac{r}{R_{1}}\right) J_{1}^{\prime \prime}\left(\alpha \Omega_{s}\right)-J_{1}\left(\Omega_{s} \frac{r}{R_{1}}\right) Y_{1}^{\prime \prime}\left(\alpha \Omega_{s}\right)\right)\right]
\end{aligned}
$$

where $Z(\omega)=d D(\omega) / d \omega$. The first term of the right-hand side is in proportion to $t$, so that the behavior of this resonant oscillation is mainly governed by the first term after a long time. When the amplitude become large, non-linearities in the Euler equations cannot be neglected any longer and the linear solution Eqn. (37) loses its validity. The corresponding fluid force takes the form of Eqn. (15) with:

$C_{a}=\frac{1}{\hat{Z}\left(\Omega_{s}\right)}\left(Y_{1}\left(\Omega_{s}\right) J_{1}\left(\alpha \Omega_{s}\right)-J_{1}\left(\Omega_{s}\right) Y_{1}\left(\alpha \Omega_{s}\right)\right)$

$C_{v}=\frac{\rho \pi R_{1}^{2} \omega_{s}}{\alpha \Omega_{s}-\left(\alpha \Omega_{s}\right)^{-1}} \frac{\omega_{s} t}{\hat{Z}\left(\Omega_{s}\right)}\left(Y_{1}\left(\Omega_{s}\right) J_{1}^{\prime}\left(\alpha \Omega_{s}\right)-J_{1}\left(\Omega_{s}\right) Y_{1}^{\prime}\left(\alpha \Omega_{s}\right)\right)$

where:

$$
\begin{aligned}
\hat{Z}\left(\Omega_{s}\right) & =\Omega_{s}\left[\frac{1-\Omega_{s}}{1-\alpha \Omega_{s}}\left(J_{1}\left(\Omega_{s}\right) Y_{1}^{\prime}\left(\alpha \Omega_{s}\right)-Y_{1}\left(\Omega_{s}\right) J_{1}^{\prime}\left(\alpha \Omega_{s}\right)\right)\right. \\
& \left.-\left(J_{1}^{\prime}\left(\Omega_{s}\right) Y_{1}\left(\alpha \Omega_{s}\right)-Y_{1}^{\prime}\left(\Omega_{s}\right) J_{1}\left(\alpha \Omega_{s}\right)\right)\right]
\end{aligned}
$$

A linearly time-dependent damping coefficient has appeared at the resonance frequency.

\section{Lubricant film models}

The challenge in lubricant film models is to include the transient and the convective inertia terms. So as to make it analytically possible, geometrical simplifications have to be made. By considering the triangle $O O^{\prime} A$ in Fig.1, the following relation is obtained:

$$
R_{1}^{2}=\left(C+R_{1}-h\right)^{2}+e^{2}-2\left(C+R_{1}-h\right) e \cos \theta
$$

The above equation is a second order polynomial in $h$. So the solution of the problem of interest is easily found:

$$
h=R_{1}+C-e \cos \theta-R_{1} \sqrt{1+\frac{C^{2}-e^{2} \sin ^{2} \theta}{R_{1}^{2}}}
$$

In a lubricant film the radial clearance $C$ and the motion amplitude $e$ are very small compared to the radius of curvature $R_{1}$ :

$$
C / R_{1}<<1 \text { and } e / R_{1}<<1
$$

Then keeping only the first order terms in Eqn. (41), the film thickness is reduced to:

$$
h(t)=C-e(t) \cos \theta
$$

Moreover, since the film is very thin, the curvature effects are often neglected in the lubrification theory. This assumption allows the prescription of a Cartesian local coordinate system $\left(x=R_{1} \theta, y\right)$ on the plane of the lubricant film. $x$ is in the direction of $\mathbf{e}_{\theta}$ and $y$ in that of $\left(-\mathbf{e}_{\mathbf{r}}\right)$ in Fig. 1. Denoting $u$ and $v$ the local fluid velocity along $x$ and $y$, the Navier-Stokes equations for an infinitely long cylindrical film becomes:

$$
\begin{aligned}
\rho\left(\frac{\partial u}{\partial t}+\frac{u}{R_{1}} \frac{\partial u}{\partial \theta}+v \frac{\partial u}{\partial y}\right) & =-\frac{1}{R_{1}} \frac{\partial p}{\partial \theta}+\mu \frac{\partial^{2} u}{\partial y^{2}} \\
\frac{\partial p}{\partial y} & =0
\end{aligned}
$$

with the mass conservation equation:

$$
\frac{\partial \rho}{\partial t}+\frac{1}{R_{1}} \frac{\partial u}{\partial \theta}+\frac{\partial v}{\partial y}=0
$$

Equation (44) is found by writing the Navier-Stokes equations in a dimensionless form and neglecting the terms of order $\left(C / R_{1}\right)^{2}$ and higher according to Eqn. (42). The boundary conditions can be written:

$$
\begin{aligned}
u=0, \quad v=0 \quad \text { at } y & =0 \\
u=U, \quad v=\frac{d h}{d t}=\frac{\partial h}{\partial t}+\frac{U}{R_{1}} \frac{\partial h}{\partial \theta} \text { at } y & =h(\theta, t)
\end{aligned}
$$

where $U(\theta, t)=-\dot{e}(t) \sin \theta$. Integrating Eqn. (45) along the fluid thickness between 0 and $h$ and using the Leibniz's integration formula gives:

$$
\frac{\partial h}{\partial t}+\frac{1}{R_{1}} \frac{\partial h \bar{u}}{\partial \theta}=0
$$

where $\bar{u}$ is the mean out-flow velocity at angle $\theta$. Integrating the above equation between 0 and $\theta$ and keeping in mind Eqn. (43) allow to obtain the expression of $\bar{u}$ without the Navier-Stokes equation:

$$
\bar{u}(t, \theta)=\frac{R_{1} \dot{e}(t) \sin \theta}{C-e(t) \cos \theta}
$$

In order to continue the analytical derivation, particular velocity profiles along the fluid thickness satisfying the boundary conditions Eqn (46) and the mean out-flow velocity Eqn. (48) are often assumed. In [8,9], a parabolic profile is used and in $[10,11]$ an el- 


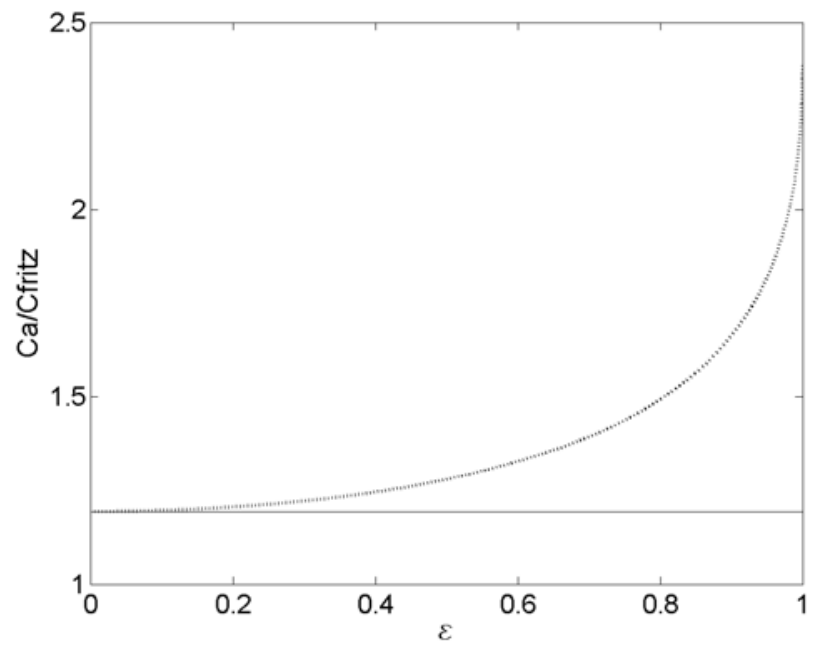

Figure 4. VARIATION OF $\frac{C_{a}^{\min }}{C_{\text {Fritz }}}$ AND $\frac{C_{a}^{\max }}{C_{\text {Fritz }}}$ WITH $\varepsilon_{m}$.

liptical one. Once the corresponding $u$ and $v$ are determined, the pressure gradients are derived with the help of Eqn. (44) by using different approximation methods (momentum, energy or iterative method). The induced forces acting on the moving cylinder take the form:

$$
\mathbf{F}(t)=-\left(\rho \pi R_{1}^{2} C_{a}(t) \ddot{e}(t)+C_{1}(t) \dot{e}(t)+C_{2}(t) \dot{e}(t)^{2}\right) \mathbf{e}_{\mathbf{x}}
$$

which is the sum of an added mass, a viscous and a convective inertia terms. The coefficients depend on the approximation methods and the assumed velocity profile. Since they vary with very similar tendencies in different papers [8,10,36,37], only the results for the energy method and parabolic velocity profile are shown here. For this case, the coefficients are given by:

$$
\begin{aligned}
& C_{a}(t)=\frac{12}{5} \frac{1}{\alpha-1} \frac{1-\sqrt{1-\varepsilon(t)^{2}}}{\varepsilon(t)^{2}} \\
& C_{1}(t)=12 \pi \mu \frac{1}{(\alpha-1)^{3}} \frac{1}{\left(1-\varepsilon(t)^{2}\right)^{3 / 2}} \\
& C_{2}(t)=\rho \pi R_{1} \frac{12}{5} \frac{1}{\alpha-1} \frac{1}{\varepsilon(t)^{2}}\left(\frac{2-\varepsilon(t)^{2}}{2 \sqrt{1-\varepsilon(t)^{2}}}-1\right)
\end{aligned}
$$

These coefficients are function of the instantaneous timedependent eccentricity ratio. For an harmonic movement, the coefficients oscillate between a maximum and a minimum value. These value for the added mass coefficients are:

$$
\begin{aligned}
C_{a}^{\min } & =\frac{6}{5} \frac{1}{\alpha-1} \\
C_{a}^{\max } & =\frac{12}{5} \frac{1}{\alpha-1} \frac{1-\sqrt{1-\varepsilon_{m}^{2}}}{\varepsilon_{m}^{2}}
\end{aligned}
$$

At a given $\varepsilon_{m}$, the added mass coefficient oscillates between these two values. The variations of $\frac{C_{a}^{\min }}{C_{\text {Fritz }}}$ and $\frac{C_{a}^{\max }}{C_{\text {Fritz }}}$ with $\varepsilon_{m}$ are shown in Fig. 4 for $\alpha=1.01$. Even with small displacements relative to radial clearance $(\varepsilon \rightarrow 0)$, it shows that the incompressible inviscid model underestimates the added mass in squeeze film flow. The difference is all the more pronounced as the confinement is high:

$$
\frac{C_{a}^{\min }}{C_{\text {Fritz }}}=\frac{6}{5} \frac{\alpha+1}{\alpha^{2}+1} \stackrel{\alpha \rightarrow 1}{\longmapsto} 1.2
$$

Figure 4 also shows that when the displacement of the inner cylinder is increased regarding the radial clearance, the maximum added mass experienced by the solid can be more than two times higher than those predicted by Fritz during an oscillation. Moreover when an elliptical velocity profile is assumed, the unsteady inertia force are roughly 1.1 to 1.2 times bigger than those presented here with the parabolic profile, and an elliptical profile is expected to better describe the effects due to large amplitude cylinder motions $[10,11]$.

\section{LIMITS AND CHALLENGES Comments on added mass}

As written by Sarpaya (2004) [38], "added mass is one of the best known, least understood, and most confused characteristics of fluid dynamics. It exists in all flows about bluff bodies. However, it manifests its existence, like all masses, only when it is accelerated". Equation (10) suggests that the fluid force can be decomposed into an inviscid inertia term and a vorticity-dependent term. However the inertia term must not be confused with the added mass. Firstly, as shown in simplified viscous models, the added mass is influenced by the viscosity, see Eqn. (26) and Eqn. (27), so that the value obtained with inviscid models can only give an idea of the added mass value. Secondly, the vorticity-dependent term contains also a part in phase with the body acceleration, therefore which must be included in the added mass. In order to be convinced, the derivation in [39] is of particular interest. A body started from rest in a fluid initially at rest is considered. During an infinitively small $\Delta t$ the vorticity produced by the incremental velocity of the cylinder is contained in a small layer $\delta$. Using the polar coordinates $(r, \theta)$ measured from the direction of acceleration $\mathbf{e}_{\mathbf{x}}$, Eqn. (10) can be rewritten:

$$
\begin{gathered}
\mathbf{F}(t)=\left(\rho \frac{d}{d t} \int_{0}^{2 \pi} I(t, \theta, \delta) \sin \theta d \theta+\rho \pi R_{1}^{2} \ddot{e}(t)\right) \mathbf{e}_{\mathbf{x}} \\
\text { with } I(t, \theta, \delta)=\int_{R_{1}}^{R_{1}+\delta}-r^{2} \omega_{z}(t, \theta) d r
\end{gathered}
$$

where $\omega_{z}$ is the vertical component of the vorticity, so that:

$$
I(t, \theta, \delta)=\int_{R_{1}}^{R_{1}+\delta}-r^{2} \frac{\partial u_{\theta}}{\partial r}-r u_{\theta}+r \frac{\partial u_{r}}{\partial \theta} d r
$$

The conditions on the body and at the edge of the singular vorticity layer can be expressed by:

$$
\begin{gathered}
\mathbf{u}\left(t, R_{1}, \theta\right)=\dot{e}(t) \cos \theta \mathbf{e}_{\mathbf{r}}-\dot{e}(t) \sin \theta \mathbf{e}_{\theta} \\
\mathbf{u}\left(t, R_{1}+\delta, \theta\right)=\dot{e}(t) \cos \theta \mathbf{e}_{\mathbf{r}}+\dot{e}(t) \sin \theta \mathbf{e}_{\theta}
\end{gathered}
$$




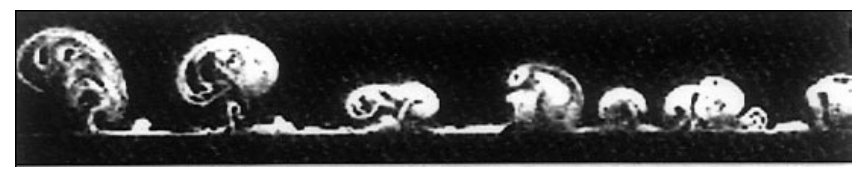

(a)

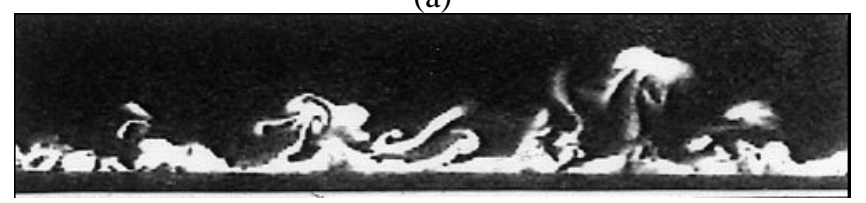

(b)

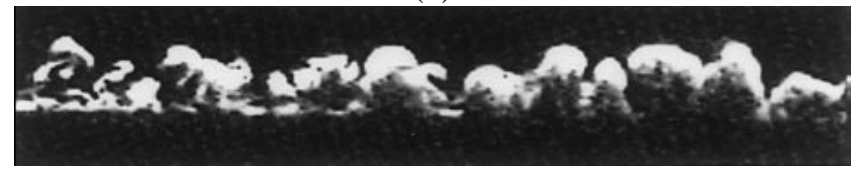

(c)

Figure 5. (a) HONJI-TYPE COHERENT STRUCTURES AT $\beta=74800$ AND $K C=0.35$ ON THE HALL-LINE, (b) QUASI-COHERENT STRUCTURES IN ZONE D AT $\beta=1365000$ AND $K C=0.3\left(1.8 K C_{c r}\right)$, (c) HTCS AND QCS IN ZONE C AT $\beta=1365000$ AND $K C=0.1$ $\left(0.59 K C_{c r}\right)$. REPRINT FROM SARPKAYA [27]

By integrating by part the first term in the integral in Eqn. (55), considering the above boundary conditions and taking the limit $\delta \rightarrow 0$, it comes:

$$
\lim _{\delta \rightarrow 0} I(\theta, \delta)=\lim _{\delta \rightarrow 0}\left[-r^{2} u_{\theta}\right]_{R_{1}}^{R_{1}+\delta}=-2 \dot{e}(t) R_{1}^{2} \sin \theta
$$

By introducing it in Eqn. (54), the fluid force is evaluated as:

$$
\mathbf{F}(t)=\left(-2 \rho \pi R_{1}^{2} \ddot{e}(t)+\rho \pi R_{1}^{2} \ddot{e}(t)\right) \mathbf{e}_{\mathbf{x}}=-\rho \pi R_{1}^{2} \ddot{e}(t) \mathbf{e}_{\mathbf{x}}
$$

which is the ideal inviscid value for an infinite fluid domain. It has been shown that if the body is started from rest in a viscous fluid initially at rest, the initial value of the added mass is in agreement with its ideal value (obtained with the potential theory) because the vorticity is still confined to a thin sheet on the boundary. In general, the diffusion has not the time to adjust to the unsteady conditions imposed on the flow, and the instantaneous state is strongly influenced by the entire history of motion. In harmonic laminar unseparated movement, this results in the creation of a damping term, and in laminar linear movement with a general time-dependency, this can be expressed by a convolution product [40]. In cases in which no wake is formed, and at sufficiently high Stokes number so that the boundary layer is very thin, this derivation can help to explain why the ideal added mass for an infinite fluid domain is a good fluid forces approximation.

\section{Stability of the two-dimensional flow}

When the inner cylinder rotates at a given frequency and the outer one is at rest, the two-dimensional fluid flow is known to be unstable to a three-dimensional centrifugal instability, resulting in the formation of the Taylor vortices. In the case consid-

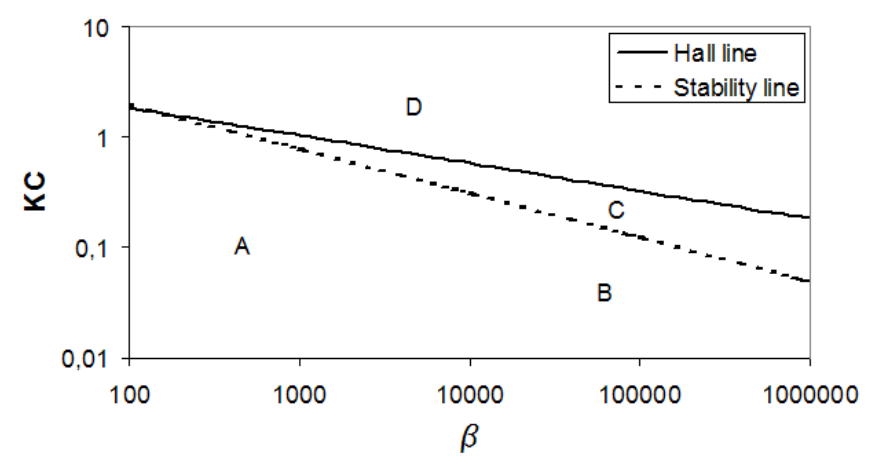

Figure 6. ZONES IN THE PARAMETERS SPACE $(K C, \beta)$.

ered in this paper, the inner cylinder is not subjected to a rotating motion, but is subjected to a radial oscillation. No result on the stability of this flow is available in the literature for a confined fluid domain. However, more is known for the case of a circular cylinder oscillating in an infinite fluid domain. The instability, which was first discovered by Honji (1981) [41], results in a well-organized "disturbed laminar" regime which has three-dimensional features. This fluid flow consists in a series of mushroom-shape vortex structures (see Fig. (5.a)) along the two lines where the local ambient velocity is maximum (at the crown of the cylinder). They are named "Honji-type coherent structures, HTCS" in [21,42]. This regime clearly provides a limit on the range of validity of the Stokes-Wang theory, which assumes a two-dimensional flow. Hall [43] carried out a stability analysis of the two-dimensional flow assuming a large $\beta$. His study shows that these coherent structures (of centrifugal nature) appear when the Keulegan-Carpenter number is equal to the critical value:

$$
K C_{c r}=5.78 \beta^{-1 / 4}\left(1+0.21 \beta^{-1 / 4}+\ldots\right)
$$

which is in accordance with Honji's experiments. This equation corresponds to the Hall-line in Fig. 6. When the KeuleganCarpenter number exceeds this critical value (zone D), HTCS may first exhibit a disturbed form (see Fig. 5(b)) giving rise to "Quasi-coherent structures, QCS" [21]. If $K C$ is again increased, separation, vortex shedding and turbulence may appeared. Irregular boundary-layer type instabilities over a finite region of $K C$ smaller than $K C_{c r}$ (zone $C$ ) are also observed [21,42]. They consist of a combination of QCS and occasional HTCS (see Fig. 5(c)). The lower limit where no instabilities are created during an entire cycle is experimentally given by the stability line defined by:

$$
K C_{s} \approx 12.5 \beta^{-2 / 5}
$$

Figure 6 displays the different zones separated by the Hall-line and the stability line. These three-dimensional structures can strongly influence fluid forces, and specially the fluid damping term. In some measurements obtained at $\beta=1000$ [5], HTCS 
are linked to a jump in the drag coefficient from $C_{d}$ (Eqn. (27)) for $K C<K C_{c r}$ to $1.3 C_{d}$ when the structures appeared. In zones A and B (Fig. 6), no instability is predicted so that the Stokes-Wang theory is expected to be valid. However, there is experimental evidence that it is only valid when $\beta<4500$. When the Stokes number exceeds this value a jump from $C_{d}$ to $2 C_{d}$ is observed in zones $\mathrm{B}$ and $\mathrm{C}$ in many experiments $[21,44,45]$. Whereas this jump could be explained by the existence of QCS in zone $\mathrm{C}$, the reason why it also occurs in zone B, even for Keulegan-Carpenter number as much as two orders of magnitude below $K C_{S}$, is still missing. This indicates that the Hall-line and the stability line are not the only limits of the Stokes-Wang theory and that the flow may be subjected to another instability not yet identified, perhaps in other regions of the cylinder. As suggested in [45], it may be induced by structural changes in the boundary layer. It will be very difficult to identify the reason of this damping term jump. Since it occurs at high Stokes number and is a three-dimensional phenomenon, it is numerically very costly and even out of reach for the highest Stokes numbers. It is also difficult to appreciate experimentally since the boundary layer is all the thinner as $\beta$ is high.

Due to the statistical nature of the structures, the different zones in Fig. 6 can not be separated by simple lines. For instance, the stability line comes from human interpretation of intermittent fluid motions [21]. Moreover, the lines depend not only on the dimensionless parameters $K C$ and $\beta$ but also on more uncontrollable ones such as the presence of air bubbles in the fluid and on the cylinder, temperature gradients, dissymmetry in the base flow, roughness. There have been some experimental attempts to identify the roughness influence in this flow. In [44], the roughness induced by an abrasive paper characterized by a ratio $k / d=0.00079$ is considered. A jump in the damping coefficient from $2 C_{d}$ for a smooth surface to $3 C_{d}$ with the abrasive paper was found. Some experiments in [21] carried out at $k / d \approx 0.01$ have shown that the flow separates from the sand grains at all the parameter values performed in the ranges $10^{4}<\beta<1.3 .10^{6}$ and $0.0052<K C<0.6$. These attempts to identified the roughness influence can not yet be industrially used since much more experiments and theoretical studies have to be performed so as to make it understandable.

\section{CONCLUSION}

Various fluid force models have been reviewed for an oscillating infinitely long circular cylinder in cylindrical confinement and in infinite fluid domain. It has been shown that the incompressible inviscid model provides a lower limit of the added mass term when the flow is unseparated. This added mass term is only slightly increased by viscous corrections, see Eqn. (26) and Eqn. (27) for a reasonable confinement, but is strongly underestimated in extreme confinement (see Eqn. (51) and Fig. 4). It is also the lower limit in case of a compressible flow, when the oscillation frequency is smaller than the resonance frequency (see
Eqn. (33) and Fig. 2). Moreover it does not predict any damping terms, which are of considerable importance even if they are much smaller than the added mass term in norm. They can effectively limit the body motions by absorbing a part of the excitation energy. Damping can arise from laminar viscous effects, see Eqn. (26) and Eqn. (27), but also from the flow induced by the body motion in lubricant film (Eqn. (50)). In infinite fluid domain, it has been shown that the damping term predicts by the Stokes-Wang theory is available only in a small part of the parameters space $(K C, \beta)$ and strongly underestimates the real damping induced by three-dimensional instabilities in the other zones. So, the simplified models used in design office tend to underestimate both added mass and damping effects. Design margin could be significantly improved by taking into account the real fluid behavior. However, a huge amount of experimental, theoretical and numerical studies is still to be done. For instance, there is nothing on the stability of the two-dimensional cylindrically confined flow induced by radial motions of the inner cylinder.

\section{REFERENCES}

[1] Bouard, R., and Coutanceau, M., 1980. "The early stage of development of the wake behind an impulsively started cylinder for $40<\operatorname{Re}<10^{4}$ ". J. Fluid Mech., 101, pp. 583607.

[2] Haller, G., 2004. "Exact theory of unsteady separation for two-dimensional flows". J. Fluid Mech., 512, pp. 257-311.

[3] Dutsch, H., Durst, F., Becker, S., and Lienhart, H., 1998. "Low-Reynolds-number flow around an oscillating circular cylinder at low Keulegan-Carpenter numbers". J. Fluid Mech., 360, pp. 249-271.

[4] Tatsuno, M., and Bearman, P., 1990. "A visual study of the flow around an oscillating circular cylinder at low Keulegan-Carpenter numbers and low Stokes numbers". J. Fluid Mech., 211, pp. 157-182.

[5] Sarpkaya, T., 1986. "Force on a circular in viscous oscillatory flow at low Keulegan-Carpenter numbers". J. Fluid Mech., 165, pp. 61-71.

[6] Williamson, C., 1985. "Sinusoidal flow relative to circular cylinders". J. Fluid Mech., 155, pp. 141-174.

[7] Koumoutsakos, P., and Leonard, A., 1995. "High resolution simulations of the flow around an impulsively started circular cylinder using vortex methods". J. Fluid Mech., 296, pp. 1-38.

[8] El-Shafei, A., and Crandall, S., 1991. "Fluid inertia forces in squeeze film dampers". ASME, Rotating Machinery and Vehicule Dynamics, 35, pp. 219-228.

[9] Tichy, J., and Bou-Saïd, B., 1991. "Hydrodynamic lubrification and bearing behavior with impulsive loads". STLE, Trib. Trans., 34, pp. 505-512.

[10] Han, Y., and Rogers, R., 2001. "Nonlinear fluid forces in 
cylindrical squeeze films. part I: short and long lengths". $J$. Fluids Struct., 15, pp. 151-169.

[11] Usha, R., and Vimala, P., 2003. "Squeeze film force using an elliptical velocity profile". ASME, J. Appl. Mech., 70, pp. 137-142.

[12] Vance, J., 1988. Rotordynamics of Turbomachinery. John Wiley and sons, New York.

[13] Sigrist, J.-F., 2004. "Modélisation et simulation numérique d'un problème couplé fluide/structure non-linéaire. Application au dimensionnement de structures nucléaires de propulsion navale". $\mathrm{PhD}$ Thesis, University of Nantes, France.

[14] Sigrist, J.-F., Laine, C., Lemoine, D., and Peseux, B., 2003. "Choice and limits of a linear fluid model for the numerical study in dynamic fluid structure interaction problem". In Proceedings of ASME, Vol. 454 of Pressure Vessel and Piping, Cleveland, pp. 87-93.

[15] Melot, V., Sigrist, J.-F., Laine, C., Auvity, B., and Peerhossaini, H., 2005. "Fluid structure interaction for a strongly accelerated cylinder: a numerical study of flow and fluid forces". In $11^{\text {th }}$ International Topical Meeting on Nuclear Reactor Thermal Hydraulics, Avignon, France.

[16] Noca, F., Shiels, D., and Jeon, D., 1999. "A comparison of methods for evaluating time-dependent fluid dynamic forces on bodies, using only velocity fields and their derivatives”. J. Fluids Struct., 13, pp. 551-578.

[17] Quartapelle, L., and Napolitano, M., 1982. "Force and moment in incompressible flows". AIAA Journal., 21, pp. 911913.

[18] Saffman, P., 1993. Vortex Dynamics. Cambridge University Press, Cambridge.

[19] Keulegan, G., and Carpenter, L., 1958. "Forces on cylinders and plates in an oscillating fluid". J. Res. Nat. Bur. Stand., 60, pp. 423-440.

[20] Axisa, F., 2001. Modélisation des systèmes mécaniques. Intéractions fluide/structure. Hermès.

[21] Sarpkaya, T., 2001. "Hydrodynamic damping and quasicoherent structures at large Stokes numbers". J. Fluids Struct., 15, pp. 909-928.

[22] White, E., Rice, J., and Ergin, F., 2005. "Receptivity of stationary transient disturbances to surface roughness". Phys. Fluids, 17, p. 064109.

[23] Bottaro, A., and Zebib, A., 1997. "Görtler vortices promoted by wall roughness". Fluid Dyn. Res., 19, pp. 343362.

[24] Joseph, G. G., Zenit, R., Hunt, M. L., and Rosenwinkel, A. M., 2001. "Particle-wall collisions in a viscous fluid". $J$. Fluid Mech., 433, pp. 329-346.

[25] Protas, B., Styczek, A., and Nowakowski, A., 2000. "An effective approach to computation of forces in viscous incompressible flows". J. Comp. Phys., 159, pp. 231-245.

[26] Tan, B., Thompson, M., and Hourigan, K., 2005. "Evaluat- ing fluid forces on bluff bodies using partial velocity data". J. Fluids Struct., 20, pp. 5-24.

[27] Morison, J., O’Brien, M., Johnson, J., and Schaaf, S., 1950. "The force exerted by surface waves on piles". Petroleum Transactions, AIME, 189, pp. 149-157.

[28] Sarpkaya, T., 2001. "On the force decompositions of Lighthill and Morison”. J. Fluids Struct., 15, pp. 227-233.

[29] Fritz, R., 1972. "The effects of liquids on the dynamics motion of immersed solids". Trans. ASME, J. Eng. Ind., pp. 163-171.

[30] Chen, S., Wambsganss, M., and Jendrzejczyk, J., 1976. "Added mass and damping of a vibrating rod in confined viscous fluids". ASME, J. Appl. Mech., pp. 325-329.

[31] Chen, S., 1987. Flow induced vibrations. Hemisphere Publishing Corporation.

[32] Sinyavaskii, V., Fedotovskii, V., and Kukhtin, A., 1980. "Oscillation of a cylinder in a viscous fluid". Prikladnaya Mekhanika, 16.

[33] Stokes, G., 1851. "On the effect of the internal friction of fuids on the motion of pendulums". Trans. Camb. Phil. Soc., 9, pp. 8-106.

[34] Wang, C., 1968. "On high-frequency oscillatory viscous flows”. J. Fluid Mech., 32, pp. 55-68.

[35] Kurihara, E., Inoue, Y., and Yano, T., 2005. "Nonlinear resonant oscillations and shock waves generated between two coaxial cylinders". Fluid Dyn. Res., 36, pp. 45-60.

[36] Lu, Y., and Rogers, R., 1995. "Instantaneous squeeze film force between a heat exchanger tube with arbitrary tube motion and a support plate". J. Fluids Struct., 9, pp. 835-860.

[37] Zhou, T., and Rogers, R., 1997. "Simulation of twodimensional squeeze film and solid contact forces acting on a heat exchanger tube". J. Sound. Vib., 203, pp. 621-639.

[38] Sarpkaya, T., 2004. "A critical review of the intrinsic of vortex-induced vibrations". J. Fluids Struct., 19, pp. 389447.

[39] Leonard, A., and Roshko, A., 2001. "Aspects of flowinduced vibration”. J. Fluids Struct., 15, pp. 415-425.

[40] Basset, A. B., 1888. "On the motion of a sphere in viscous fluid”. Phil. Trans. Roy. Soc. Lond., 179, pp. 43-63.

[41] Honji, H., 1981. "Streaked flow around an oscillating circular cylinder". J. Fluid Mech., 107, pp. 507-520.

[42] Sarpkaya, T., 2002. "Experiments on the stability of sinusoidal flow over a circular cylinder". J. Fluid Mech., 457, pp. 157-180.

[43] Hall, P., 1983. "On the stability of unsteady boundary layer on a cylinder oscillating transversely in a viscous flow". $J$. Fluid Mech., 146, pp. 337-367.

[44] Chaplin, J., and Subbiah, K., 1998. "Hydrodynamic damping of a cylinder in still water and in transverse current". Appl. Ocean Res., 20, pp. 251-259.

[45] Chaplin, J., 2000. "Hydrodynamic damping of a cylinder at $\beta=10^{6}$ ". J. Fluids Struct., 14, pp. 1101-1117. 